\title{
Descomposición de hojarasca de Pinus radiata y tres especies arbóreas nativas
}

\author{
Decomposition of leaf litter of Pinus radiata and three native tree species
}

\author{
CHRISTOPHER H. LUSK ${ }^{1}$, CESAR DONOSO ${ }^{1}$, MYLTHON JIMÉNEZ¹, CAROLINA MOYA¹, GISELA OYARCE¹, RODRIGO \\ REINOSO $^{1}$, ALFREDO SALDAÑA ${ }^{1}$, PAOLA VILLEGAS $^{1} \&$ FRANCISCO MATUS $^{2}$ \\ ${ }^{1}$ Departamento de Botánica, Universidad de Concepción, Casilla 160-C, Concepción, Chile, \\ e-mail: clusk@udec.cl \\ ${ }^{2}$ Departamento de Producción Agrícola, Universidad de Talca, Talca, Chile
}

\begin{abstract}
RESUMEN
En el centro-sur de Chile, las últimas décadas han sido testigo de una conversión masiva de bosques, matorrales y tierras agrícolas a plantaciones de árboles exóticos. Aunque se ha estudiado la influencia de dichos cambios sobre el balance hídrico, los posibles efectos sobre otros procesos ecosistémicos han recibido poca atención. En esta breve comunicación se presentan datos de un estudio de la descomposición de hojarasca de Pinus radiata y tres especies arbóreas nativas, llevado a cabo con el fin de explorar los posibles efectos del reemplazo de bosque nativo por plantaciones exóticas sobre el ciclaje de nutrientes. Se incubaron muestras de las cuatro especies en dos ambientes distintos en sitios colindantes: bajo un bosque nativo secundario, y bajo un rodal de $P$. radiata. Se registró la pérdida de peso seco después de dos meses y seis meses. Las tasas diarias de descomposición fueron mucho mayores durante los primeros dos meses de incubación que durante los cuatro meses subsiguientes. En ambas fechas hubo diferencias significativas entre las especies y entre los sitios: todas las especies presentaron mayores tasas de descomposición bajo $P$. radiata que bajo el bosque nativo. No hubo evidencia de interacción entre sitio y especie. Después de seis meses, el orden de pérdida de peso seco fue Nothofagus obliqua $>$ P. radiata $>$ Peumus boldus $>$ Cryptocarya alba. La variación interespecífica en la tasa de descomposición presentó más relación con el área foliar específica que con el contenido de nitrógeno en la hojarasca. Dado que la hojarasca de $P$. radiata se descompuso más lentamente que la de la especie caducifolia $N$. obliqua, pero más rápidamente que las especies esclerófilas, los efectos de la sustitución o invasión sobre descomposición dependerían de la composición original del bosque nativo en cuestión.
\end{abstract}

Palabras clave: área foliar específica, ciclaje de nutrientes, nitrógeno foliar, Nothofagus, plantaciones exóticas.

\begin{abstract}
Recent decades have seen widespread conversion of native forests, shrublands and farmland in south-central Chile to exotic tree plantations. However, little is known about the effects of these wholesale landuse changes on ecosystem properties and processes, with the notable exception of studies of site water balance. In this brief communication, we present the results of a comparative study of decomposition of leaf litter of Pinus radiata and three common native tree species, beneath exotic and native woody vegetation in south-central Chile. We aimed to assess the nutrient cycling implications of substitution or invasion of native vegetation by $P$. radiata. Litter samples of the four species were incubated in both environments, registering the percentage of dry weight loss after two and six months. Decomposition rates of all species were much faster during the first two months of incubation than during the four subsequent months. At both dates there were significant differences between species and between sites, with faster decomposition of all species beneath $P$. radiata. There was no evidence of interaction between species and site. After six months, species rank order for the percentage of weight loss was Nothofagus obliqua $>$ P. radiata $>$ Peumus boldus $>$ Cryptocarya alba. Interspecific variation in decomposition rates was more closely correlated with specific leaf area than with litter nitrogen content. Given that litter of $P$. radiata decomposed slower than that of the deciduous $N$. obliqua, but faster than the sclerophyll evergreens, the consequences of substitution or invasion for decomposition processes are likely to depend on the composition of the native vegetation in question.
\end{abstract}

Key words: exotic plantations, leaf nitrogen, Nothofagus, nutrient cycling, specific leaf area.

\section{INTRODUCCIÓN}

La conversión de grandes extensiones del centro-sur de Chile a plantaciones de árboles exóticos ha generado inquietudes sobre las posibles consecuencias ambientales de dichos cambios (Lara \& Veblen 1993). Aparte del impacto evidente sobre la diversidad biológica, en los últimas años se ha estudiado la influencia de las plantaciones exóticas sobre el balance hídrico del sitio, y el rendimiento hídrico de las 
cuencas (e.g., Huber 1992'1, Otero et al. 1994). Sin embargo, los posibles efectos de estos cambios sobre otros procesos y propiedades ecosistémicos aún han recibido poca atención. Los efectos de las especies introducidas de Pinus son especialmente dignos de atención, dada la comprobada capacidad de algunas especies de este género de invadir vegetación natural y seminatural en los países del hemisferio sur (Richardson et al. 1994). Por lo tanto, los pinos introducidos tienen el potencial de alterar las propiedades ecosistémicas no sólo en los sectores asignados a plantaciones, sino también en la vegetación nativa intervenida y susceptible a la invasión (Versfeld \& Van Wilgen 1986).

Las diferencias interespecíficas en las propiedades de la hojarasca inciden en los procesos locales de descomposición, y por lo tanto en el ciclaje de carbono y nutrientes minerales (Lambers et al. 1998). Propiedades de la hojarasca asociadas a una tasa lenta de descomposición incluyen una alta razón C:N (Lambers et al. 1998), una alta razón de lignina a nutrientes minerales (Melillo et al. 1982), y una abundancia de fenoles u otros compuestos secundarios (Cornelissen 1996). Una descomposición lenta reduce la disponibilidad de nutrientes para el nuevo crecimiento, debido a la inmovilización de estos en las acumulaciones de detritus. Por otra parte, una descomposición rápida de la hojarasca, producto de una menor razón $\mathrm{C}: \mathrm{N}$, un bajo contenido de lignina o escasez de compuestos secundarios, potencia un ciclaje más rápido de los nutrientes en el ecosistema forestal. Aunque los ambientes bióticos y abióticos sin duda influyen en las tasas de descomposición, no se sabe con certeza hasta que punto la tasa de descomposición es afectado por la especialización de la fauna detritívora a la vegetación autóctona de una región. Hansen (1999) encontró que la descomposición de muestras de hojarasca de Quercus rubra en un bosque norteamericano fue potenciada por detritívoros especialistas, naturalmente asociados a esta especie. Por otra parte, un estudio comparativo reciente de las tasas de descomposición de la hojarasca de un gran número de angiospermas Argentinas demostró que el orden de las especies fue robusto frente la variación ambiental (Pérez-Harguindeguy et al. 2000).

En otros países, se ha documentado que algunas especies de coníferas tienden a producir grandes acumulaciones de detritus, a causa de una descomposición lenta (Vogt et al. 1986, Dames et al.
1998, Silvester \& Orchard 1999). Dado que la primera rotación de $P$. radiata en Chile crece sobre suelos que se han desarrollado bajo otros tipos de vegetación (bosques y matorrales nativos, praderas), si la tasa de descomposición de la hojarasca de $P$. radiata difiriese sustancialmente a la de la vegetación anterior, durante rotaciones sucesivas se esperaría ver alteraciones en la disponibilidad de nutrientes, y por lo tanto, en la productividad del sitio. En este artículo, documentamos las tasas de descomposición en terreno de la hojarasca de $P$. radiata y de tres de las especies arbóreas nativas más abundantes del centro-sur de Chile: Nothofagus obliqua (Mirb.) Oerst, Cryptocarya alba (Mol.) Looser y Peumus boldus Mol. Con el fin de estudiar los efectos tanto de la especie como del ambiente sobre las tasas de descomposición, se incubaron muestras de las cuatro especies bajo un bosque nativo secundario y bajo un rodal de $P$. radiata, ubicados en sitios colindantes.

\section{MATERIALES Y MÉTODOS}

\section{Sitio de estudio}

El estudio se realizó en la localidad de Santa Juana ( $\left.37^{\circ} 11^{\prime} \mathrm{S}, 72^{\circ} 55^{\prime} \mathrm{O}\right), 50 \mathrm{~km}$ al sur de Concepción. La hojarasca fue colectada e incubada en dos ambientes: un bosque nativo secundario, dominado por Nothofagus obliqua y Cryptocarya alba, y un rodal de Pinus radiata de 10 años de edad, regenerado espontáneamente después de la tala de una plantación anterior de 30 años de edad. Por lo tanto, el segundo sitio había sido ocupado continuamente por $P$. radiata durante 40 años. Se taladraron cuatro de los árboles más grandes en el bosque nativo, y el mayor tenía ca. 100 anillos de crecimiento. Ambos sitios habían sido cultivados durante varios siglos antes del reestablecimiento de una cobertura forestal. Ambos sitios se hallaron en pendientes moderadas de exposición oriente, de $20-25^{\circ}$ en el caso de $P$. radiata, y de 25-30 ${ }^{\circ}$ para el bosque nativo. Suelos francos profundos $(>70 \mathrm{~cm})$ estaban presentes en ambos sitios. Con el fin de evaluar la posible influencia de la densidad del dosel forestal sobre regímenes térmicos y tasas de descomposición, se utilizó un analizador de doseles LAI-2000 (Li-Cor Inc. Lincoln, Nebraska) para medir el porcentaje de apertura del dosel en 15 puntos aleatorios en cada sitio.

\section{Incubaciones}

Durante una semana en abril 1999, se recolectó hojarasca de $P$. radiata y de las tres especies más
HUBER A (1992) Redistribución de las precipitaciones y balance hídrico de un rodal de Pinus radiata y un bosque nativo en el sur de Chile. Actas de Segundo Simposio Pinus radiata: investigación en Chile, Valdivia, Chile. 6 pp. 
comunes del bosque nativo estudiado (Nothofagus obliqua, Cryptocarya alba, Peumus boldus) sobre trampas de malla sombradera. La hojarasca fue separada por especie, y muestras de 2,0 g (peso seco) fueron selladas en bolsas de malla sombradera, con apertura de 2,5 x 2,0 mm. Esta apertura permite el acceso al interior de las bolsas por un rango de detritívoros invertebrados, pero minimiza las pérdidas por fragmentación (Douce \& Crossley 1982). Al comienzo de mayo 1999, se enterraron ocho réplicas de cada especie en la capa de hojarasca en cada sitio, a una profundidad de unos dos centímetros. Se amarraron las bolsas a la base del árbol más próximo para evitar su extravío. Después de dos meses (comienzo de julio 1999), se recuperaron cuatro réplicas para determinar la pérdida de peso seco. Después de seis meses (comienzo de noviembre 1999) se recuperaron las cuatro réplicas restantes.

La pérdida de peso seco durante la descomposición no es un proceso lineal, sino obedece a una cibernética de primer orden (Matus \& Rodríguez 1994):

$$
\mathrm{y}=\mathrm{A}_{\mathrm{o}} \cdot \mathrm{e}^{-\mathrm{k} \cdot \mathrm{t}}
$$

donde $y=$ el peso seco restante, $A_{o}=$ peso seco inicial, $\mathrm{t}=$ tiempo. Por lo tanto, además de medir las pérdidas absolutas de peso seco, se calcularon las tasas diarias de pérdida de peso. La siguiente ecuación sirvió para obtener una tasa no lineal de primer orden:

$$
\Delta \mathrm{y}=\ln \mathrm{y}_{2}-\ln \mathrm{y}_{1} / \mathrm{t}_{2}-\mathrm{t}_{1}
$$

Con la finalidad de explorar las relaciones de la estructura y química foliar con la variación

\section{TABLA 1}

Características de la hojarasca de las cuatro especies estudiadas (promedio $\pm \mathrm{EE}$ ).

Promedios seguidos por la misma letra no difieren significativamente (prueba HSD de Tukey-Kramer, $\mathrm{P}=0,05)$

Leaf litter traits for the four study species (mean \pm SE). Means followed by the same letter do not differ significantly at $\mathrm{P}=0.05$ (Tukey-Kramer HSD test)

\begin{tabular}{lcr}
\hline Especie & $\begin{array}{c}\text { Nitrógeno total } \\
(\%)\end{array}$ & $\begin{array}{c}\text { Área foliar } \\
\text { específica } \\
\left(\mathrm{cm}^{2} \mathrm{~g}^{-1}\right)\end{array}$ \\
\hline Pinus radiata & $0,62 \pm 0,07 \mathrm{a}$ & $71,6 \pm 7,0 \mathrm{a}$ \\
Nothofagus obliqua & $0,54 \pm 0,06 \mathrm{a}, \mathrm{b}$ & $109,1 \pm 7,5 \mathrm{~b}$ \\
Peumus boldus & $0,52 \pm 0,05 \mathrm{a}, \mathrm{b}$ & $65,5 \pm 1,7 \mathrm{a}$ \\
Cryptocarya alba & $0,40 \pm 0,04 \mathrm{~b}$ & $64,0 \pm 1,4 \mathrm{a}$ \\
\hline
\end{tabular}

interespecífica en las tasas de descomposición, se midieron el área foliar específica (AFE) y el contenido total de nitrógeno de la hojarasca (Tabla 1). Se determinó el AFE mediante la medición del área proyectada de muestras con un areafoliómetro Li-COR 3000, dividiendo dicha área por el peso seco. El N total se determinó por el método Kjeldahl, en el Laboratorio de Análisis de Suelos y Plantas, Universidad de Concepción.

\section{Análisis estadísticos}

Se utilizó análisis de varianza (ANDEVA) multifactorial para determinar los efectos de especie y ambiente sobre las tasas de descomposición de la hojarasca. Mediante correlaciones se examinaron las relaciones de la variación interespecífica en las tasas de descomposición con el área foliar específica y el $\mathrm{N}$ total de la hojarasca, transformando logarítmicamente los datos de AFE para linearizar la relación. Se realizaron todos los análisis con JMP Statistical Software (SAS Inc.).

\section{RESULTADOS}

Después de dos meses de incubación, la pérdida promedio de peso seco varió entre un $16 \%$ para Cryptocarya alba y 23,5\% para Nothofagus obliqua (Fig. 1A). El ANDEVA indicó que tanto la especie como el ambiente incidieron significativamente en las tasas de descomposición (Tabla 2), con mayores tasas bajo el rodal de $P$. radiata (Fig. 1A). No hubo evidencia de interacción entre especie y ambiente (Tabla 2). Las únicas diferencias significativas entre especies individuales fueron aquellas separando $C$. alba de $N$. obliqua y P. boldus (prueba HSD de Tukey-Kramer, $\mathrm{P}<0,05)$.

Después de seis meses de incubación, la pérdida promedio de peso seco varió entre un $25,2 \%$ para C. alba y un $40,2 \%$ para $N$. obliqua (Fig. 1B). No hubo correlación significativa entre la variación interespecífica de pérdida de peso seco a los dos meses, y la a los seis meses $(\mathrm{r}=0,80, \mathrm{P}$ $=0,20)$, y las posiciones de $P$. boldus y $P$. radiata se invirtieron entre las dos fechas (Fig. 1). El ANDEVA nuevamente arrojó efectos significativos de especie y de ambiente sobre las tasas de descomposición, pero no se detectó ninguna evidencia de interacción entre especie y ambiente (Tabla 2). La pérdida de peso seco de N. obliqua después de seis meses fue significativamente mayor que la de $P$. boldus y C. alba, y la pérdida promedio para $P$. radiata también fue 


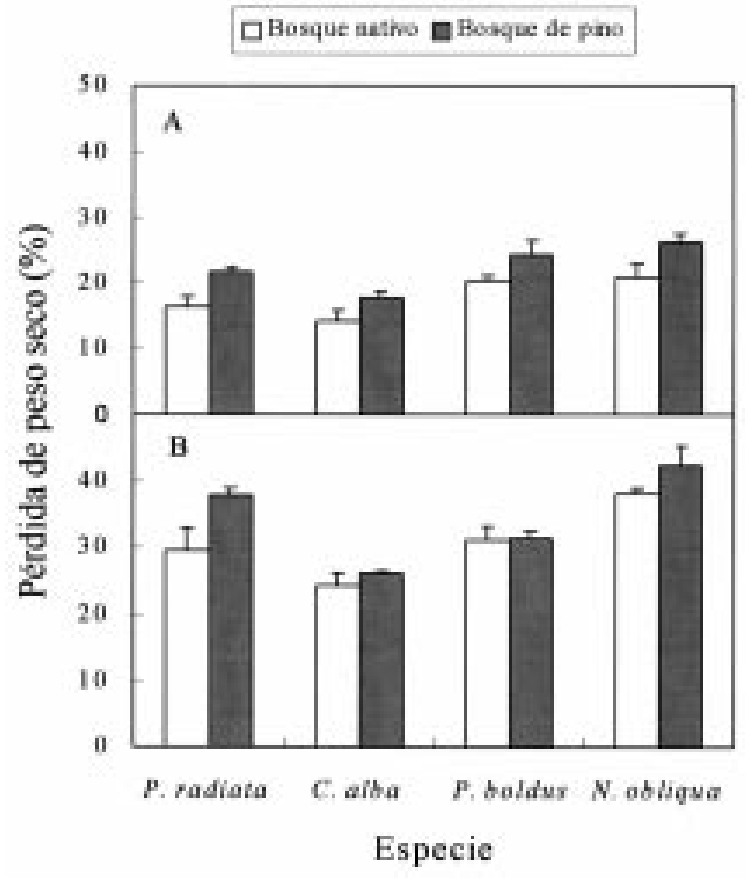

Fig. 1: Pérdida de peso seco de hojarasca, luego de (A) dos meses y (B) seis meses de incubación bajo bosque nativo y bajo un rodal de Pinus radiata. Barras indican un error estándar.

Dry weight loss by leaf litter samples incubated for (A) two months and (B) six months beneath native forest and beneath a stand of $P$. radiata. Bars show one standard error.

significativamente mayor que la de $C$. alba (prueba HSD de Tukey-Kramer, $\mathrm{P}<0,05$ ).

Las muestras de todas las especies perdieron más peso absoluto durante los dos primeros meses de incubación que durante los cuatro meses subsiguientes (Fig. 1). Además, las tasas diarias de descomposición ( $\mathrm{mg} \mathrm{g}^{-1}$ día $^{-1}$ ) de todas las especies fueron claramente mayores durante los primeros dos meses de incubación que durante los cuatro meses subsiguientes (Fig. 2).

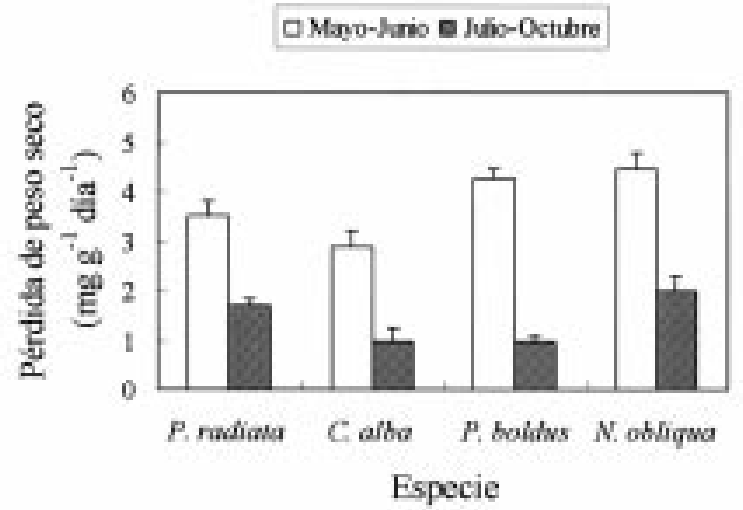

Fig. 2: Tasas diarias de descomposición de la hojarasca de cuatro especies arbóreas durante los primeros dos meses de incubación (mayo-junio) y durante los últimos cuatro meses (julio-octubre). Barras indican un error estándar. Para toda especie, las tasas diarias fueron significativamente mayores (prueba t de Student, $\mathrm{P}<0,0001$ ) durante los primeros dos meses que durante los últimos cuatro meses.

Average daily decomposition rates of leaf litter of four tree species during the first two months (May-June) and last four months (July-October) of incubation. Bars show standard error. For all species, daily rates during the first two months were significantly higher (Student t-test, $\mathrm{P}<$ 0.0001 ) than those during the last four months.

A los dos meses, la variación interespecífica en la pérdida de peso seco no se correlacionó fuertemente ni con el $(\log )$ AFE $(r=0,69, \mathrm{P}=0,32) \mathrm{ni}$ con el $n$ total $(r=0,51, P=0,49)$. A los seis meses, tampoco hubo correlaciones significativas, aunque el porcentaje de pérdida de peso seco presentó más relación con el $(\log )$ AFE $(r=0,90, \mathrm{P}=$ $0,10)$ que con el nitrógeno total $(\mathrm{r}=0,71, \mathrm{P}=$ $0,29)$.

TABLA 2

Efectos de especie y ambiente sobre pérdida de peso seco de muestras de hojarasca, después de dos y seis meses de incubación

Environment and species effects on percent dry weight loss of leaf litter samples, after two and six months' incubation

\begin{tabular}{|c|c|c|c|c|c|}
\hline \multirow[b]{2}{*}{ Fuente de variación } & \multirow[b]{2}{*}{ Grados de libertad } & \multicolumn{2}{|c|}{ Dos meses } & \multicolumn{2}{|c|}{ Seis meses } \\
\hline & & Valor de F & Valor P & Valor de F & Valor P \\
\hline Especie & 3 & 10,93 & 0,0001 & 18,85 & $<0,0001$ \\
\hline Ambiente & 1 & 18,92 & 0,0002 & 7,08 & 0,0143 \\
\hline Especie $\mathrm{x}$ ambiente & 3 & 0,18 & 0,9066 & 1,76 & 0,185 \\
\hline
\end{tabular}


El porcentaje de apertura ( \pm error estándar) del dosel fue significativamente mayor bajo el rodal de pino $(13,4 \pm 0,1)$ que bajo el bosque nativo $(8,8$ $\pm 0,8)$ (prueba t de Student, $\mathrm{P}=0,0033$ ).

\section{DISCUSIÓN}

Las tasas diarias de descomposición fueron mucho mayores durante los primeros dos meses de incubación que durante los cuatro meses subsiguientes (Fig. 2). Patrones semejantes han sido documentados en otros estudios de descomposición de hojarasca (e.g., Melillo et al. 1982, Cornelisson 1996). Aunque dicha variación podría explicarse parcialmente por las diferencias de temperatura entre el otoño y los meses de invierno y primavera, también es factible que se deba a una degradación rápida de los componentes más lábiles de la hoja, tales como los carbohidratos solubles, durante las fases iniciales de la descomposición (Suberkropp et al. 1976).

Después de seis meses, la hojarasca de Pinus radiata se había descompuesto más que la de las especies nativas siempreverdes Cryptocarya alba y Peumus boldus, aunque menos que la de la especie nativa caducifolia Nothofagus obliqua (Fig. 1B). Por lo tanto, los efectos precisos de una sustitución o invasión por $P$. radiata podrían depender de la composición original del bosque nativo en cuestión. Es de esperar que la sustitución o invasión de un bosque dominado por especies caducifolias de Nothofagus provoque un retardo en las tasas de descomposición. Por el contrario, el reemplazo de bosque esclerófilo por $P$. radiata debería acelerar las tasas de descomposición. En el caso de un bosque mixto de Nothofagus caducifolio y especies esclerófilas, probablemente no habría mayor alteración en las tasas de descomposición, y por ende, en el ciclaje de nutrientes, ya que $P$. radiata presenta tasas de descomposición intermedias entre $N$. obliqua y las especies esclerófilas. En el único estudio comparable de que tenemos conocimiento, se encontró que la hojarasca de plantaciones de $P$. radiata en la isla sur de Nueva Zelanda se descompuso a una tasa similar a la de bosques nativos dominados por especies siempreverdes de Nothofagus (Heng 1980).

Nuestros resultados sugieren que la hojarasca de $P$. radiata es más descomponible que la de algunos de sus congéneres que han sido plantadas comercialmente en otros países. Por ejemplo, Springett (1976) documentó menores tasas de descomposición, y menores densidades de detritívoros bajo plantaciones de $P$. pinaster in Australia occidental que bajo la vegetación nativa adyacente. Así mismo, se han encontrado acumulaciones masivas de hojarasca no descompuesta en plantaciones de $P$. patula en Sudáfrica (Dames et al. 1998) y de $P$. ponderosa en Nuevo México (Vogt et al. 1986). Estas diferencias entre $P$. radiata y ciertas otras especies del género pueden reflejar el contenido nutricional relativamente alto de la hojarasca de $P$. radiata (Vogt et al. 1986), y su bajo grado de esclerofilia, comparado con otros pinos.

Las diferencias interespecíficas en la pérdida de peso seco después de seis meses fueron marginalmente correlacionadas con la variación en el área foliar específica (AFE), pero no con la concentración de nitrógeno en la hojarasca. El AFE es a menudo un buen indicador de las tasas de descomposición de hojarasca (Lambers et al. 1998). Aunque dicha correlación podría atribuirse en parte a la influencia de la razón de área superficial a volumen sobre las tasas de descomposición, sin duda alguna también juega la relación inversa del AFE con la inversión en defensas físicas y químicas, tales como lignina y otros compuestos fenólicos (Lambers et al. 1998). Aunque su valor adaptativo principal es la prolongación de la vida funcional de la hoja (Coley et al. 1985), estas defensas también retardan la descomposición (Cornelisson 1996, Lambers et al. 1998).

Además de la variación interespecífica en las tasas de decomposición, el ANDEVA también identificó un efecto significativo del ambiente: muestras incubadas bajo $P$. radiata presentaron mayor pérdida de peso seco que aquellas enterradas bajo el bosque nativo, en ambas fechas de muestreo (Fig. 1). Esta diferencia podría explicarse por una razón C:N más favorable en el piso del rodal de $P$. radiata, debido a la alta concentración de $\mathrm{N}$ en la hojarasca de esta especie (Tabla 1). Por otra parte, mayor llegada de radiación solar al piso, como resultado del mayor grado de apertura del dosel, podría acelerar la descomposición bajo $P$. radiata.

Estudios de la fauna detritívora en el centro-sur de Chile indican que la composición y diversidad de ésta varía notoriamente entre distintos tipos de cobertura boscosa (Martínez \& Casanueva 1993, 1995). Sin embargo, nosotros no encontramos evidencia que el ambiente de incubación actuara de forma diferencial sobre las tasas de descomposición de las cuatro especies, ya que el ANDEVA no detectó interacción significante entre especie y ambiente (Tabla 2). Por tanto, nuestros resultados, implicando cierta equivalencia funcional entre distintas faunas detritívoras, apoyan las conclusiones del trabajo extenso realizado por Pérez-Harguindeguy et al. (2000), que la variación interespecífica en las tasas de descomposición no es afectada mayormente por el ambiente. 


\section{LITERATURA CITADA}

COLEY PD, JP BRYANT \& FS CHAPIN (1985) Resource availability and plant anti-herbivore defence. Science 230: 895-899.

CORNELISSEN JHC (1996) An experimental comparison of leaf decomposition rates in wide range of temperate plant species and types. Journal of Ecology 84: 573582.

DAMES JF, MC SCHOLES \& CJ STRAKER (1998) Litter production and accumulation in Pinus patula plantations of the Mpumalanga Province, South Africa. Plant and Soil 203: 183-190.

DOUCE GK \& DA CROSSLEY (1982) The effect of soil fauna on litter mass loss and nutrient dynamics in arctic tundra at Barrow, Alaska. Ecology 63: 523537.

HANSEN RA (1999) Red oak litter promotes a microarthropod functional group that accelerates its decomposition. Plant and Soil 209: 37-45.

HENG S (1980) The addition, decomposition and accumulation of organic matter in some native Nothofagus forests and Pinus radiata plantations in the South Island of New Zealand. Tesis de Doctorado (Ph.D.), Lincoln College, University of Canterbury, Lincoln, New Zealand.

LAMBERS H, FS CHAPIN \& TL PONS (1998) Plant physiological ecology. Springer-Verlag, New York, New York. 540 pp.

LARA A \& TT VEBLEN (1993) Forest plantations in Chile: a successful model? En: Mather A (ed) Afforestation policies, planning and progress: 118139. Belhaven Press, London, United Kingdom.

MARTÍNEZ RI \& ME CASANUEVA (1993) Ácaros Oribátidos del alto Bío-Bío, Chile: diversidad y abundancia relativa (Acari: Oribatida). Gayana Zoología (Chile) 57: 7-19.

MARTÍNEZ RI \& ME CASANUEVA (1995) Fauna oribatológica de Chile: nuevo registro de especies humícolas en las regiones VIII y IX (Acari, Oribatida). Boletín de la Sociedad de Biología de Concepción (Chile) 66: 43-51.

Editor Asociado: J. Armesto

Recibido el 10 de julio de 2000; aceptado el 24 de mayo de 2001
MATUS FJ \& J RODRÍGUEZ (1994) A simple model for estimating the contribution of nitrogen mineralization to the nitrogen supply of crops from a stabilized pool of soil organic matter and recent organic input. Plant and Soil 162: 259-271.

MELILLO JM, JA ABER \& JF MURATORE (1982) Nitrogen and lignin control of hardwood leaf descomposition. Ecology 63: 621-626.

OTERO L, A CONTRERAS \& L BARRALES (1994). Efectos ambientales del reemplazo de bosque nativo por plantaciones (estudio en cuatro microcuencas en la provincia de Valdivia). Ciencia e Investigación Forestal (Chile) 8: 253-276.

PÉREZ-HARGUINDEGUY $\mathrm{N}, \mathrm{S}$ DÍAZ, JHC CORNELISSEN, F VENDRAMINI, M CABIDO \& A CASTELLANOS (2000) Chemistry and toughness predict leaf litter decomposition rates over a wide spectrum of functional types and taxa in central Argentina. Plant and Soil 218: 21-30.

RICHARDSON DA, PA WILLIAMS \& RJ HOBBS (1994) Pine invasions in the southern hemisphere: determinants of spread and invadibility. Journal of Biogeography 21: 511-527.

SILVESTER WB \& TA ORCHARD (1999) The biology of kauri (Agathis australis) in New Zealand. I. Production, biomass, carbon storage and litterfall in four forest remnants. New Zealand Journal of Botany 37: 553-571.

SPRINGETT JA (1976) The effect of planting Pinus pinaster on populations of soil microarthropods and on litter decomposition at Gnangara, western Australia. Australian Journal of Ecology 1: 83-87.

SUBERKROPP K, GL GODSHALK \& MJ KLUG (1976) Changes in the chemicalcomposition of leaves during processing in a woodland stream. Ecology 57: 720727.

VERSFELD DB \& BW VAN WILGEN (1986) Impacts of woody aliens on ecosystems properties. En: MacDonald IAW (ed) The ecology and control of biological invasions in South Africa: 239-246. Oxford University Press, Cape Town, South Africa.

VOGT KA, CG GRIER CC \& DJ VOGT (1986) Production, turnover and nutrient dynamics of above- and belowground detritus of world forests. Advances in Ecological Research 15: 303-377. 